Document downloaded from:

http://hdl.handle.net/10251/169193

This paper must be cited as:

Zavala-Alcívar, A.; Verdecho Sáez, MJ.; Alfaro Saiz, JJ. (2020). Assessing and Selecting Sustainable and Resilient Suppliers in Agri-Food Supply Chains Using Artificial Intelligence: A Short Review. IFIP Advances in Information and Communication Technology. 598:501510. https://doi.org/10.1007/978-3-030-62412-5_41

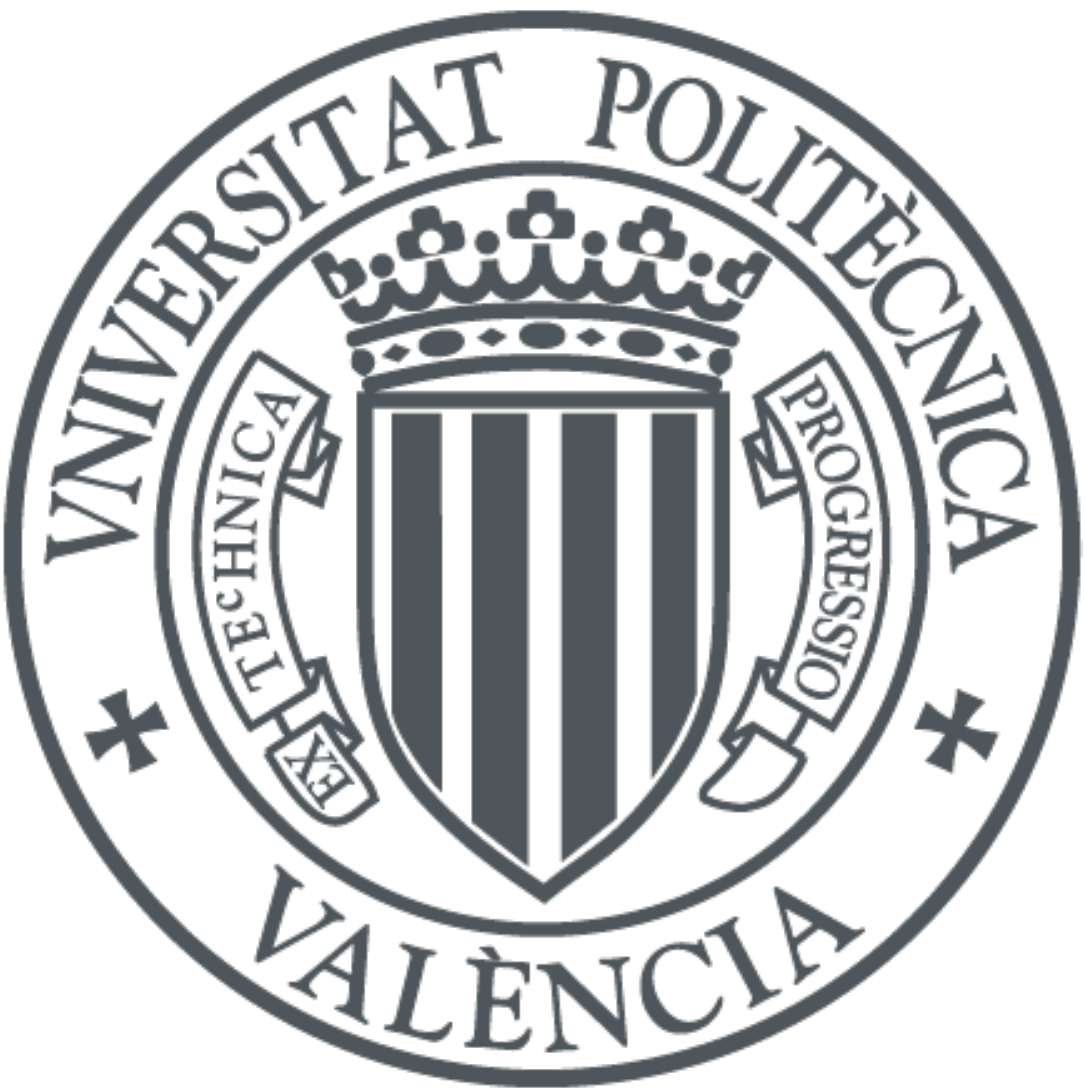

The final publication is available at

https://doi.org/10.1007/978-3-030-62412-5_41

Copyright Springer

Additional Information 


\title{
Assessing and selecting sustainable and resilient suppliers in agri-food supply chains using artificial intelligence: a short review
}

\author{
Antonio Zavala-Alcívar ${ }^{1}$, María-José Verdecho² and Juan-José Alfaro-Saiz² \\ ${ }^{1}$ Faculty of Industrial Engineering, Universidad Laica Eloy Alfaro de Manabí, Manta \\ 130214, Ecuador \\ ${ }^{2}$ Research Centre on Production Management and Engineering (CIGIP), Universitat \\ Politècnica de València, Camino de Vera S/N, Valencia, 46022 Spain \\ antonio.zavala@uleam.edu.ec; mverdecho@cigip.upv.es; jalfaro@cigip.upv.es
}

\begin{abstract}
The supplier evaluation and selection process is critical to increase the sustainability and resilience of the agri-food supply chain. Therefore, in this sector, it is necessary to consider sustainability and resilience criteria in the supplier evaluation and selection process. The use of artificial intelligence techniques allows managing of a lot of information and the reduction of uncertainty for decision making. The objective of this article is to analyze articles that address the selection of suppliers in agrifood supply chains that pursue to increase their sustainability and resilience by using artificial intelligence techniques to analyze the techniques and criteria used and draw conclusions.
\end{abstract}

Keywords: Artificial intelligence, supplier selection, sustainability, resilience, agri-food supply chains.

\section{Introduction}

The supplier evaluation and selection process is one of the fundamental processes in supply chain management. In global markets, there are multiple characteristics that make this process complex such as a greater number of potential suppliers, changing procurement regulations, modification of social policies, constant change in customer preferences, complex decision making when operating in the scope of the supply chain, etc. These characteristics imply that decision making must be quick, adaptive, and complex due to the consideration of uncertainty and, multiple criteria and stakeholders to be satisfied [1].

The criteria generally demanded to suppliers are quality, price, delivery time, and service [2]. In addition, if supply chains are to be resilient and sustainable, it is necessary to consider those criteria that allow increasing business continuity in the face of an interruption in operations [3] and, in turn, increase the sustainability of the chain [4], [5]. 
This challenge is greater in some types of supply chains, for example, in agri-food supply chains, where their characteristics, including the management of perishable products [6], require, among others, rapid and efficient decision-making. Therefore, the evaluation and selection criteria for suppliers must reflect these needs and techniques must be used that allow managing of a lot of information and the reduction of uncertainty [7], which is why artificial intelligence techniques are appropriate.

The objective of this paper is to analyze articles that discuss models regarding the sustainable and resilient supplier selection process in agrifood supply chains using artificial intelligence techniques.

The structure of this paper is as follows. First, the parameters of the search are defined. Then, the analysis of the articles is performed in three points: evolution over time, artificial intelligence techniques and supplier selection criteria. Finally, conclusions are exposed.

\section{Literature Review}

Supplier evaluation and selection research started in the early 1960s [8] and remains a topic of high-interest today because of its impact on supply chain management. In the current context, it is necessary to include in this process criteria that increase the sustainability and resilience of the supply chain. Several articles have analyzed the main criteria [7] and the main methods and techniques applied in the literature for the evaluation and selection of suppliers. The research [9] analyses 170 articles and concludes that the main techniques for supplier selection are multi-criteria techniques (MCDM), mathematical programming, and artificial intelligence techniques. An analysis of the main MCDM methods used for the process is provided in [10]-[12] Recent studies have examined hybrid techniques and methods to provide evaluation and selection with more specific criteria [13].

[11] indicate that the focus of supplier evaluation and selection has been extended to a scenario where qualitative, economic, and environmental performance are optimized. Sustainability criteria are structured in three dimensions: economic, environmental, and social. Resilient supply chain criteria can be grouped into four types [14], [15]: supply chain re-engineering, collaboration, agility, and culture. Although recent studies incorporate a greater number and variety of criteria to assess the sustainability of suppliers [3], [4], [13], [16], there are few studies dedicated to the assessment and selection of suppliers jointly with resilience criteria [4].

The applications of artificial intelligence have been a growing topic of interest in the last years. Factors such as managing of large amounts of data shared information, and data processing and predicting for effective decision-making by all members, make the application of artificial intelligence increasingly used [17]. Besides, characteristics such as perishability, seasonality, and changing weather conditions in the agri-food sector [6], make this sector a field for the implementation of artificial intelligence techniques. 


\subsection{Search criteria}

This article has developed the methodological approach of the Systematic Literature Review (SLR) proposed by [18], which aims to evaluate and interpret available and relevant research on a research question, thematic area, or phenomenon of interest. This approach starts with the formulation of research questions, literature location, selection, analysis and synthesis of papers, and the reporting of results.

Given the objective of this work, the following research question is addressed: What artificial intelligence techniques and criteria have been used for the evaluation and selection of sustainable and resilient suppliers in the agri-food supply chain?

Several studies have classified the main artificial intelligence techniques for supplier evaluation and selection. In this article, based on the classifications proposed by [9], [12] and [13], the following techniques are considered: Genetic Algorithm (GA), Neural Networks (NN), Case-based reasoning (CBR), Grey system theory (GST), Rough set theory (RST), Fuzzy Logic (FL), Bayesian Networks (BN), Differential Evolution (DE), Particle Swarm Optimization (PSO).

Due to the variety of artificial intelligence techniques, several search keywords were established by changing the artificial intelligence technique used. For genetic algorithms the search string was: ("partner" OR "supplier" OR "vendor") $A N D$ ("selection" OR "evaluation" OR "performance" OR "monitoring" OR "development" OR "assessment" OR "approaches") AND ("agri-food" OR "food" OR "agriculture*" OR "agribusiness") AND ("genetic algorithm").

The database used for the search was Scopus. Initially, 24 articles were obtained. Then, the results were filtered using the following criteria: English language, peerreviewed indexed journals, and time range from the year 2000. This produced 11 articles, which are analyzed below.

\subsection{Result and discussion}

\subsubsection{Temporal analysis}

From the results obtained, it can be observed that the topic is a field of recent research, with increasing interest. The first articles were published in 2010, in which two articles were published (Table 1). So far, in 2020, the largest number of articles have been published, 4 articles, which reflects a recent interest in incorporating artificial intelligence techniques for the selection of suppliers in the agri-food sector.

\subsubsection{Artificial Intelligence Techniques Analysis}

Table 1 presents the main artificial intelligence techniques used in the articles as well as whether only one technique is applied in the solution (Single), several techniques (Hybrid), and whether the study makes a comparison between techniques (Single comparative). The table also presents the uncertainty mitigation approaches applied in the papers analyzed. For this purpose, the proposal by Hamdi et al. (2018) was considered, establishing three approaches to model risk and uncertainty for supplier selection: fuzzy, stochastic and others [20]. The percentage values are presented at the bottom of the table. 
It should be noted that no articles were found that used the Case-based Reasoning and Differential Evolution techniques, although they have been used in other sectors [21]-[23]. The main techniques used are Fuzzy Logic, Genetic Algorithm, and Rough Set Theory. Most of the articles combine two or more artificial intelligence techniques, i.e. the use of hybrid techniques is extended to provide the advantages of different techniques in the proposed solution.

Table 1. Principal artificial intelligence techniques in supplier evaluation and selection for agri-food supply chains.

\begin{tabular}{|c|c|c|c|c|c|c|c|c|c|c|c|c|c|c|}
\hline \multirow{2}{*}{$\begin{array}{c}\text { Articl } \\
\mathrm{e}\end{array}$} & \multirow[b]{2}{*}{ Year } & \multicolumn{7}{|c|}{ Artificial Intelligence Techniques } & \multicolumn{3}{|c|}{ Application type } & \multicolumn{3}{|c|}{ Uncertainty } \\
\hline & & GA & $\mathrm{NN}$ & GST & RST & FL & $\mathrm{BN}$ & PSO & Single & Hybrid & $\begin{array}{c}\text { Single } \\
\text { Comparative }\end{array}$ & Fuzzy & Stochastic & $\begin{array}{c}\text { Other } \\
\mathrm{s}\end{array}$ \\
\hline$[24]$ & 2010 & & & & $\sqrt{ }$ & & & & $\checkmark$ & & & & & $\checkmark$ \\
\hline$[25]$ & 2010 & & & $\checkmark$ & $\checkmark$ & & & & & $\checkmark$ & & & & $\checkmark$ \\
\hline$[26]$ & 2013 & & $\checkmark$ & & $\checkmark$ & & & & & $\checkmark$ & & $\checkmark$ & & \\
\hline$[27]$ & 2015 & & & & & $\checkmark$ & & & $\checkmark$ & & & $\checkmark$ & & \\
\hline$[28]$ & 2017 & $\checkmark$ & & & & & & & $\checkmark$ & & & & $\checkmark$ & \\
\hline [29] & 2018 & $\checkmark$ & & & & $\checkmark$ & & $\checkmark$ & & $\checkmark$ & $\checkmark$ & $\checkmark$ & $\checkmark$ & \\
\hline$[30]$ & 2019 & $\checkmark$ & & & & & $\checkmark$ & & & $\checkmark$ & & $\checkmark$ & & \\
\hline$[31]$ & 2020 & & & & & $\checkmark$ & & & $\checkmark$ & & & $\checkmark$ & & \\
\hline$[32]$ & 2020 & & & & & $\checkmark$ & & & $\checkmark$ & & & $\checkmark$ & & \\
\hline [33] & 2020 & & & & & $\checkmark$ & $\checkmark$ & & & $\checkmark$ & & $\checkmark$ & $\checkmark$ & \\
\hline [34] & 2020 & $\checkmark$ & & & & & & $\checkmark$ & & $\checkmark$ & $\checkmark$ & & $\checkmark$ & \\
\hline & $\begin{array}{c}\text { Total } \\
\%\end{array}$ & $\begin{array}{c}4 \\
36, \\
7\end{array}$ & $\begin{array}{c}1 \\
9,0 \\
9\end{array}$ & $\begin{array}{c}1 \\
9,09\end{array}$ & $\begin{array}{c}3 \\
27,3\end{array}$ & $\begin{array}{c}5 \\
45 \\
5\end{array}$ & $\begin{array}{c}2 \\
18 \\
2\end{array}$ & $\begin{array}{c}2 \\
18,2\end{array}$ & $\begin{array}{c}5 \\
45,5\end{array}$ & $\begin{array}{c}6 \\
54,5\end{array}$ & $\begin{array}{c}2 \\
18,2\end{array}$ & $\begin{array}{c}7 \\
63,6\end{array}$ & $\begin{array}{c}4 \\
36,7\end{array}$ & $\begin{array}{c}2 \\
18,2\end{array}$ \\
\hline
\end{tabular}

The most used technique is FL (5 articles). Articles [27], [29], [31]-[33] apply the FL technique to set the level of importance or weight of the criteria for supplier evaluation and selection. This allows them to incorporate uncertainty in the criteria to improve efficiency in the selection of suppliers. Secondly, AG is used in four articles: [28]-[30], [34]. AG facilitates selection by generating multiple solutions and adapting decision-makers' assessments to changing environments.

Thirdly, the RST technique is used in 3 articles [24]-[26] characterized by analyzing information acquired basically by experience in an objective way. This technique was the first to be used, but, since 2013, no publications were found in the agri-food sector. BN and PSO have been used in two articles. Studies using BN techniques [30], [33] organize the criteria for evaluating and selecting suppliers into a set of variables and the inter-dependency relationships between them. This technique allows us to establish the probability of unknown variables based on known ones. The PSO articles [29], [34] make it possible to develop a simple interaction between the agents (supplier-manufacturer relationship) by allowing them to interact and adapting their behavior. This characteristic allows PSO techniques to be used not only for supplier evaluation and selection but also for order allocation in changing 
environments. These two techniques (BN and PSO) have been used in conjunction with other techniques.

The GST and NN techniques are used in one article. [25] uses the GST technique because it allows problems to be solved under uncertainty with discrete data and incomplete information. The research considers the advantage of the method that it does not require knowledge of the distribution of parameters or complex intermediate models. [26] applies the NN technique that allows it to estimate or approximate functions that may depend on a large number of inputs. These techniques are also used in conjunction with other techniques to improve their effectiveness.

The main feature of the articles analyzed is the combination of different artificial intelligence techniques to reduce uncertainty and improve efficiency in decision making. Six articles consider a hybrid model and, two articles carry out comparative studies and five articles use a simple technique. The temporal evolution in the application of the techniques can also be analyzed. RST was the first to be applied individually, then in hybrid models together with GST and NN. The FL and GA techniques are applied in many cases jointly. Finally, the BN and PSO techniques have been applied recently.

Two papers develop a comparison between techniques [29], [34]. [29] concludes that the analysis of statistical and unidirectional variance and hypothesis testing show that both algorithms are relatively equal, and the results of the two algorithms do not differ significantly. [34] concludes that the techniques used generated a near-optimal solution in a very short time that did not differ from each other, even when considering multiple agents in several periods.

Considering the approaches to model risk and uncertainty for supplier selection, seven papers employ fuzzy set theory to incorporate inherent uncertainty into the decision model [26], [27], [29]-[33]. Within these papers, techniques such as fuzzy analytical hierarchy process, fuzzy multi-objective mathematical programming [27] or fuzzy DEMATEL [32] are used. Four papers apply stochastic modelling [28], [29], [33], [34] based on scenario analysis. This approach accepts randomness and uses stochastic problem parameters to reduce uncertainty. Two papers belong to the Others class. In this case, quantitative risk measures are used to mitigate uncertainty [24], [25]. Finally, two papers apply the fuzzy and stochastic approaches to reduce uncertainty, generating comparisons in their applied artificial intelligence techniques [29], [33].

\subsubsection{Criteria for supplier evaluation and selection}

In the supplier evaluation and selection process, there has been a change in the criteria used to face new requirements. The first works only considered classic business criteria such as cost, quality, etc. The complexity of decision making within the supply chain demanded the introduction of other types of criteria [25] that consider collaboration, working together towards sustainability, and generating resilience in the face of disruptive events. The importance of the criteria lies in their joint and interrelated analysis that allows for the generation of a supplier evaluation and selection process following the strategic objectives and supply chain performance [5].

Considering the analysis of the articles and the criteria for the evaluation and selection of suppliers for sustainable and resilient supply chains established by [4], 
[13], [16], [35], [36], a classification of criteria into four groups is proposed: business, collaboration, sustainability and resilience. The business criteria are those traditionally used in the supplier evaluation and selection process and cover the criteria: cost/price (C/P), quality (Q), flexibility (F), delivery (D), financial situation (FS) and product characteristics (PC). Collaboration criteria are criteria that have recently been used to measure the degree of coherence between different organizations working or wishing to work collaboratively [37]. These criteria include [4], [14], [38]: technology capability (TC), information shared (IS), process/strategic alignment (PA), culture (C), trust (T). The sustainability criteria cover two (environmental and social) of the three dimensions of sustainability (economic, environmental and social) as economic criteria have been classically considered in the supplier evaluation and selection literature and already appear within the business criteria. The sustainability criteria therefore comprise [4], [13], [16], [39]: pollution practices (PP), employment practices (EP), reputation (R), health and safety practices (HSP) and stakeholder's involvement (SKI). Finally, resilience criteria measure the aspects that impact on improving the resilience of supply chains and include [4], [5], [36]: responsiveness/velocity (RV), geographic segregation (GS), redundancy (surplus inventory) (RSI), rerouting (RE), risk reduction plan (RRP), customization $(\mathrm{C})$, visibility (V), and market adaptation (MA). It has to be noted that criteria are inter-related. For example, the criteria classified in the collaborative group allow an increase in the resilience and sustainability of the supply chain. However, this type of analysis is out of the scope of this paper. Table 2 shows the distribution of the articles in each group.

Table 2. Principal criteria for supplier evaluation and selection in the agri-food supply chain

\begin{tabular}{c|cccccc|ccccc|ccc|cccc}
\hline \multirow{2}{*}{ Article } & \multicolumn{4}{|c|}{ Business } & \multicolumn{4}{c|}{ Collaboration } & \multicolumn{3}{|c|}{ Sustainable } & \multicolumn{3}{c}{ Resilient } \\
\hline & C/P & Q & D & F & FS & PC & TC & IS & PA & C & T & PP & EP & HSP & RV & GS & RSI & RE \\
\hline$[24]$ & $\checkmark$ & $\checkmark$ & $\checkmark$ & $\checkmark$ & & $\checkmark$ & & & & $\checkmark$ & & $\checkmark$ & & & $\checkmark$ & & $\checkmark$ \\
{$[25]$} & $\checkmark$ & $\checkmark$ & $\checkmark$ & & $\checkmark$ & & & & & $\checkmark$ & & $\checkmark$ & $\checkmark$ & $\checkmark$ & & & & \\
{$[26]$} & $\checkmark$ & $\checkmark$ & $\checkmark$ & $\checkmark$ & $\checkmark$ & $\checkmark$ & & $\checkmark$ & & $\checkmark$ & & $\checkmark$ & $\checkmark$ & $\checkmark$ & $\checkmark$ & & $\checkmark$ & $\checkmark$ \\
{$[27]$} & $\checkmark$ & $\checkmark$ & $\checkmark$ & $\checkmark$ & & $\checkmark$ & $\checkmark$ & & & $\checkmark$ & & $\checkmark$ & $\checkmark$ & $\checkmark$ & & & & \\
{$[28]$} & & & $\checkmark$ & & & & & & $\checkmark$ & & & $\checkmark$ & & $\checkmark$ & & $\checkmark$ & & $\checkmark$ \\
{$[29]$} & $\checkmark$ & $\checkmark$ & $\checkmark$ & & $\checkmark$ & $\checkmark$ & & & & & & & & & & & $\checkmark$ & \\
{$[30]$} & $\checkmark$ & $\checkmark$ & $\checkmark$ & $\checkmark$ & $\checkmark$ & $\checkmark$ & $\checkmark$ & $\checkmark$ & & $\checkmark$ & & $\checkmark$ & & & $\checkmark$ & $\checkmark$ & $\checkmark$ & $\checkmark$ \\
{$[31]$} & $\checkmark$ & $\checkmark$ & $\checkmark$ & & & & $\checkmark$ & & & & $\checkmark$ & & & & $\checkmark$ & & & $\checkmark$ \\
{$[32]$} & $\checkmark$ & & & $\checkmark$ & & & & & & $\checkmark$ & & $\checkmark$ & $\checkmark$ & $\checkmark$ & $\checkmark$ & & $\checkmark$ & $\checkmark$ \\
{$[33]$} & & $\checkmark$ & $\checkmark$ & & $\checkmark$ & $\checkmark$ & & & & & & & & & & & \\
{$[34]$} & $\checkmark$ & $\checkmark$ & & & & & & & & & & & & & & & & \\
\hline
\end{tabular}

The criteria mostly used for supplier evaluation and selection are those belonging to the business criteria group, being the main criteria price, quality, delivery and flexibility. It should be noted that the financial situation is the criterion least often considered for the selection of suppliers, despite its relevance. In the group of collaboration criteria, the main criterion is culture and to a lesser extent the criteria: 
technological capacity, information shared, trust and strategic alignment. Lack of considering these criteria reduces the supply chain's capability to integrate business objectives and coordinate the way actions are implemented throughout the system [11].

Concerning sustainability criteria, the three criteria that have been used in agrifood chains are: pollution practices, employment practices, and health and safety practices [24]-[27], [32]. On the other hand, the criteria of reputation and stakeholder's involvement have not been used. The last group concerns resilience criteria. The main criteria analyzed focus on the efficient response and opportunity of the supplier (responsiveness/velocity, redundancy, rerouting) in a reactive sense and allowing an efficient and fast response when a disruptive event in the supply chain is generated. Other criteria that promote prevention capacity in the supply chain [15], [16]: risk reduction plan, customization, visibility, and market adaptation were not considered by the articles despite their relevance, especially in the agri-food sector where their characteristics generate a lot of uncertainty in operations [26].

Comparing the papers in Table 1 and Table 2, several points can be observed. Collectively, the articles using fuzzy logic have considered all business and sustainability criteria in their analysis. But individually, no paper was found that integrated all business and sustainability criteria into its analysis. Collaboration and resilience criteria are little considered in fuzzy logic models. In addition, information shared, process/strategic alignment and geographic segregation have not been considered in these papers [27], [29], [31]-[33]. Articles using rough set theory do not consider all the criteria for collaboration and sustainability. Their analysis does not include technology capability, process/strategic alignment, trust and geographic segregation [24]-[26]. The supplier assessment and selection process using a genetic algorithm integrates most criteria, except trust and employment practices [28]-[30], [34]. Papers using Bayesian Networks integrate all business and resilience criteria, but exclude from the analysis the criteria of process/strategic alignment, trust, employment practices, reputation, health and safety practices [30], [33]. Collaboration criteria are the least considered when papers use the Neural Networks technique. It has not been considered technology capability, process/strategic alignment, trust and geographic segregation [26]. The articles with Particle Swarm Optimization and Grey system theory are the ones that integrate the least number of criteria. PSO does not consider any of the collaboration and sustainability criteria, and only considers redundancy (surplus inventory) within the resilience criteria [29], [34]. GST does not consider resilience criteria and only considers culture of the collaboration criteria [25].

Papers using fuzzy set theory have considered most of the criteria in their analysis. [26], [27], [29]-[33]. By contrast, articles using stochastic modeling have focused only on business criteria. They have not considered technology capability, information shared, culture, trust, employment practices and responsiveness/velocity. [28], [29], [33], [34]. Finally, papers with quantitative risk measures to mitigate uncertainty do not integrate the collaboration and resilience criteria as technology capability, information shared, process/strategic alignment, trust, geographic segregation and rerouting [29], [33]. 


\section{Conclusion}

Supplier assessment and selection is a key process to increase agri-food supply chain sustainability and resilience. In the literature, although few applications use artificial intelligence techniques to support this process, most applications are published recently showing an increasing interest in the last years. The artificial techniques most used are fuzzy logic and genetic algorithms, being hybrid techniques the most used. Some papers also deal with the comparison of two single techniques.

The analysis of literature reviews in supplier selection uses different types of criteria that in this paper are grouped with four types: business, collaboration, sustainability and resilience criteria. When analyzing the papers, it is observed that business criteria are most used, but collaboration, sustainability and resilience criteria should be further considered if agri-food supply chains desire to increase their competitiveness. The papers reviewed use some of the criteria but do not integrate in their models the different types of criteria within each group. Thus, more applications are needed that cover a wide selection of criteria so that the supplier assessment and selection process can be enhanced.

Artificial intelligence techniques are used for different purposes in agri-food supply chains. The process of supplier assessment and selection is one of the applications. More applications are needed that increase the development of suppliers over time. Thus, the supplier assessment and selection process can be extended in a dynamic manner incorporating the performance evaluation, segmentation and improvement of suppliers over time. For that purpose, artificial intelligence techniques could be used for example to classify and forecast future performance of suppliers.

\section{Acknowledgements}

Authors of this publication acknowledge the contribution of the Project 691249, RUC-APS "Enhancing and implementing Knowledge based ICT solutions within high Risk and Uncertain Conditions for Agriculture Production Systems" (www.rucaps.eu), funded by the European Union under their funding scheme H2020-MSCARISE-2015.

\section{References}

1. Brandenburg, M., Govindan, K., Sarkis, J., Seuring, S.: Quantitative models for sustainable supply chain management: Developments and directions. Eur. J. Oper. Res. 233, 299--312 (2014)

2. Ocampo, L.A., Abad, G.K.M., Cabusas, K.G.L., Padon, M.L.A., Sevilla, N.C.: Recent approaches to supplier selection: A review of literature within 2006-2016. Int. J. Integr. Supply Manag. 12, 22--68 (2018)

3. Valipour, S., Safaei, A.: A resilience approach for supplier selection: Using Fuzzy Analytic Network Process and grey VIKOR techniques. J. Clean. Prod. 161, 431-451 (2017) 
4. Amindoust, A.: A resilient-sustainable based supplier selection model using a hybrid intelligent method. Comput. Ind. Eng. 126, 122--135 (2018)

5. Zavala-Alcívar, A., Verdecho, M.-J. and Alfaro-Saiz, J.-J.: A Conceptual Framework to Manage Resilience and Increase Sustainability in the Supply Chain. Sustainability, 12, 16, 6300 (2020).

6. Villalobos, J.R., Soto-Silva, W.E., González-Araya, M.C., González-Ramirez, R.G.: Research directions in technology development to support real-time decisions of fresh produce logistics: A review and research agenda. Comput. Electron. Agric. 167. (2019)

7. Ristono, A., Pratikto, Budi Santoso, P., Pambudi Tama, I.: A literature review of design of criteria for supplier selection. J. Ind. Eng. Manag. 11, 680--696 (2018)

8. Torres-Ruiz, A., Ravindran, A.R.: Multiple criteria framework for the sustainability risk assessment of a supplier portfolio. J. Clean. Prod. 172, 4478--4493. (2018)

9. Setak, M., Sharifi, S., Alimohammadian, A.: Supplier selection and order allocation models in supply chain management: A review. World Appl. Sci. J. 18, 55--72 (2012)

10. Ravindran, A.R., Warsing, D.P.: Supplier selection models and methods. In: Supply Chain Engineering: Models \& Applications. Taylor \& Francis Group, Boca Raton, Florida (2013)

11. De Boer, L., Labro, E., Morlacchi, P: A review of methods supporting supplier selection. Eur. J. Purch. Supply Manag. 7, 75--89 (2011)

12. De Felice, F., Deldoost, M.H., Faizollahi, M., Petrillo, A.: Performance measurement model for the supplier selection based on AHP. Int. J. Eng. Bus. Manag. 7, 1--13 (2015)

13. Zimmer, K., Fröhling, M., Schultmann, F.: Sustainable supplier management - a review of models supporting sustainable supplier selection, monitoring and development. Int. J. Prod. Res. 54, 1412--1442 (2016)

14. Christopher, M., Peck, H.: Building the Resilient Supply Chain. Int. J. Logist. Manag. 15, $1--14$ (2014)

15. Ali, A., Mahfouz, A., Arisha, A.: Analysing supply chain resilience: integrating the constructs in a concept mapping framework via a systematic literature review. Supply Chain Manag. 22, 16--39 (2017)

16. Verdecho, M.-J., Alarcón-Valero, F., Pérez-Perales, D., Alfaro-Saiz, J.-J., RodríguezRodríguez, R.: A methodology to select suppliers to increase sustainability within supply chains. Cent. Eur. J. Oper. Res. (2020)

17. Rabelo, L., Bhide, S., Gutierrez, E.: Artificial intelligence: Advances in research and applications, Artificial Intelligence: Advances in Research and Applications. Nova Science Publishers, Inc., Department of Industrial Engineering and Management Systems, University of Central Florida, Orlando, FL, United States (2017)

18. Denyer, D., Tranfield, D.: Producing a systematic review., in: The Sage Handbook of Organizational Research Methods. SAGE Publications Ltd, pp. 671--689 (2019)

19. Chen, Y.-J.: Structured methodology for supplier selection and evaluation in a supply chain. Inf. Sci. (Ny). 181, 1651--1670 (2011)

20. Hamdi, F., Ghorbel, A., Masmoudi, F., and Dupont, L.: Optimization of a supply portfolio in the context of supply chain risk management: literature review. J. Intell. Manuf., 29, 4, 763--788, (2018)

21. Kumar, V., Srinivasan, S., Das, S.: Optimal solution for supplier selection based on SMART fuzzy case base approach, in: 2014 Joint 7th International Conference on Soft Computing and Intelligent Systems, SCIS 2014 and 15th International Symposium on Advanced Intelligent Systems, ISIS 2014. Institute of Electrical and Electronics Engineers Inc., Department of Computer Science, IISJ Yokohama, Tokai Chiba, Japan, pp. 386--391. (2014)

22. Jahani, A., Azmi Murad, M.A., bin Sulaiman, M.N., Selamat, M.H.: An agent-based supplier selection framework: Fuzzy case-based reasoning perspective. Strateg. Outsourcing 8, 180--205 (2015) 
23. Wang, Q.: Hybrid knowledge-based flexible supplier selection, in: 8th International Conference on Management of E-Commerce and e-Government, ICMeCG 2014. Institute of Electrical and Electronics Engineers Inc., Department of Information Management, Shanghai Finance University, Shanghai, China, pp. 235--239 (2014)

24. Bai, C., Sarkis, J.: Green supplier development: Analytical evaluation using rough set theory. J. Clean. Prod. 18, 1200--1210 (2010)

25. Bai, C., Sarkis, J.: Integrating sustainability into supplier selection with grey system and rough set methodologies. Int. J. Prod. Econ. 124, 252--264 (2010)

26. Guo, F., Lu, Q.: Partner selection optimization model of agricultural enterprises in supply chain. Adv. J. Food Sci. Technol. 5, 1285--1291 (2013)

27. Azadnia, A.H., Saman, M.Z.M., Wong, K.Y.: Sustainable supplier selection and order lotsizing: An integrated multi-objective decision-making process. Int. J. Prod. Res. 53, 383--408 (2015)

28. Miranda-Ackerman, M.A., Azzaro-Pantel, C., Aguilar-Lasserre, A.A.: A green supply chain network design framework for the processed food industry: Application to the orange juice agrofood cluster. Comput. Ind. Eng. 109, 369--389 (2017)

29. Hajikhani, A., Khalilzadeh, M., Sadjadi, S.J.: A fuzzy multi-objective multi-product supplier selection and order-allocation problem in supply chain under coverage and price considerations: An urban agricultural case study. Sci. Iran. 25, 431--449 (2018)

30. Zhang, H., Cui, Y.: A model combining a Bayesian network with a modified genetic algorithm for green supplier selection. Simulation 95, 1165--1183 (2019)

31. Yadav, S., Garg, D., Luthra, S.:Selection of third-party logistics services for internet of things-based agriculture supply chain management. Int. J. Logist. Syst. Manag. 35, 204--230. (2020)

32. Yazdani, M., Wang, Z.X., Chan, F.T.S.: A decision support model based on the combined structure of DEMATEL, QFD and fuzzy values. Soft Comput. (2020)

33. Zhang, H., Feng, H., Cui, Y., Wang, Y.: A fuzzy Bayesian network model for quality control in O2O e-commerce. Int. J. Comput. Commun. Control 15 (2020)

34. Salehi Amiri, S.A.H., Zahedi, A., Kazemi, M., Soroor, J., Hajiaghaei-Keshteli, M.: Determination of the optimal sales level of perishable goods in a two-echelon supply chain network. Comput. Ind. Eng. 139 (2020)

35. Roy S., et al., A framework for sustainable supplier selection with transportation criteria. Int. J. Sustain. Eng. 13,2, 77--92 (2020)

36. Valipour Parkouhi, S., Safaei Ghadikolaei, A. and Fallah Lajimi, H.: Resilient supplier selection and segmentation in grey environment. J. Clean. Prod. 207, 1123--1137 (2019)

37. Camarinha-Matos, L.M., Afsarmanesh, H., Galeano, N., Molina, A.: Collaborative networked organizations - Concepts and practice in manufacturing enterprises. Comput. Ind. Eng. 57, 46--60 (2009)

38. Lezoche, M., Panetto, H., Kacprzyk, J., Hernandez, J., Alemany Díaz, M.: Agri-food 4.0: A survey of the Supply Chains and Technologies for the Future Agriculture. Comput. Ind. 117, 103187 (2020)

39. Alikhani, R., Torabi, S. and Altay, N.: Strategic supplier selection under sustainability and risk criteria. Int. J. Prod. Econ. 208, 69-82 (2019). 